\title{
EZH2 and cancer stem cells: fact or fiction?
}

\section{"...the prognostic role of $\mathrm{EZH} 2$ in different cancer types has been confirmed by several independent studies."}

\section{KEYWORDS: cancer stem cells $\approx$ cell culture $\approx$ EZH2 $\approx$ polycomb $\approx$ serum}

Several recent publications have shed new light on the role of epigenetic modifications in cancer stem cell (CSC) biology. Among other epigenetic players, polycomb group genes appear to be particularly attractive. Indeed, they are emerging as CSC-specific markers, as well as potential targets for cancer therapy. However, before these discoveries can be applied to the clinical setting, the specific role of polycomb group genes in CSC tumorigenic activity should be explained further.

Polycomb group genes are a set of epigenetic regulators that catalyze specific histone posttranslational modifications [1]. They are organized in multimeric polycomb repressive complexes (PRCs). Among them, PRC2 has caught the attention of many cancer scientists. PRC2 is responsible for histone $\mathrm{H} 3$ Lys27 methylation. In normal stem cells, this epigenetic mark contributes to lineage-specific gene silencing and self renewal [2]. The EZH2 locus encodes for the catalytic subunit of PRC2. Varambally and co-workers first demonstrated that $E Z H 2$ may be an oncogene [3]. In prostate cancer cells, EZH2 silencing reduces proliferation and invasion. In addition, EZH2 is overexpressed in metastatic versus localized prostate cancer and predicts a poor prognosis. Since then, publications on EZH2 and cancer have grown exponentially, reaching more than 250 papers published in 8 years.

Owing to the role of EZH2 in stem cell biology and cancer, a common goal has been to identify the function of EZH2 in CSCs. As known, CSCs are a rare subgroup of cancer cells with peculiar features: illimited self-renewal potential, tumorigenicity and chemoresistance [4]. CSCs can activate abnormal differentiation pathways, thereby generating several types of differentiated cancer cells, which make up the bulk of the tumor mass. Differentiated cancer cells are generally more susceptible than CSCs to chemoradiotherapy.
The CSC hypothesis is very intriguing, since it predicts that only CSCs are responsible for recurrence after therapy. Eradicating CSCs may completely inhibit tumor progression. Thus, the identification of CSC-specific genes is a hot topic in cancer research.

Following this line of reasoning, it is obvious to want to determine if EZH2 is a CSC-specific gene, or if it acts as an oncogene for all cancer cells. For some cancer types, like prostate and brain tumors, EZH2 seems to play a crucial role in CSC biology. Both genetic and pharmacological EZH2 inhibition is able to eradicate glioblastoma CSC self renewal and tumorigenicity [5]. In addition, high EZH2 expression predicts a poor prognosis and is associated with a higher grade glioma [6,7]. A whole-genome expression analysis identified genes more likely to be correlated with EZH2 expression in brain tumors [7]. Interestingly, these genes belonged to categories such as cell cycle, embryonic development and mitotic roels of polo-like kinases. Polo kinases are involved in neural stem cell symmetric division [8], thereby establishing a further link between EZH2, cancer and stem cell biology. Similarly, EZH2 is upregulated in prostate CSCs and a PRC2 repressive signature predicts poor prognosis in metastatic prostate cancer patients [9]. Unpublished data from our group confirms that EZH2 targeting selectively eradicates prostate CSCs. In addition, EZH2 activation is essential for prostate cancer metastatic spreading [10], evidence confirming the theory that CSCs are the seeds of metastasis [11].

Despite this evidence, the EZH2-CSC link has been questioned by recent work, comparing EZH2 expression between differentiated cancer cells and CSCs [12]. Surprisingly, the authors found that EZH2 expression and global levels of histone H3 Lys 27 methylation were significantly lower in CSCs. This was coupled to a global DNA

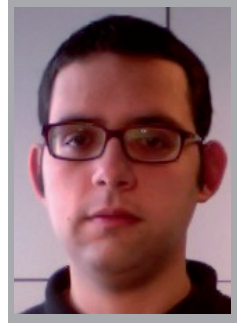

Francesco Crea Department of Internal Medicine, Division of Pharmocology, University of Pisa, Italy Tel.: +393473908226 Fax: +390502218758 fcrea@sssup.it 
demethylation in CSCs, compared with differentiated cancer cells. These experiments have been conducted on just two cell lines: MCF7 (breast cancer) and HUH7 (hepatocarcinoma). It would be interesting to test EZH2 expression in other cell lines, particularly in prostate and brain tumors, where the CSC-specificity is well established. In addition, it is well known that CSC gene-expression profile is highly dependent on culture medium composition. Culturing CSC in serum-containing media may induce CSC differentiation and completely abolish their self-renewal ability [13]. According to the papers 'materials and methods' section, Yasuda and co-workers cultured cells in Dulbecco's modified Eagle's medium plus 10\% fetal bovine serum. This regular culture medium may have affected CSC differentiation status and the epigenetic profile. Thus, these provoking results need to be repeated on a larger number of cell lines and with different culture media.

In general, testing the clinical importance of the CSC hypothesis will need a functional approach [14]. To study the role of a CSC gene in a specific tumor, pharmacological inhibiton and genetic silencing are crucial. For example, the functional importance of EZH2 in brain CSCs has been tested by both genetic and pharmacological targeting [5]. These data are more informative than a simple gene-expression comparison and pave the way to the clinical application of EZH2 inhibitors. In addition, the prognostic role of EZH2 in different cancer types has been confirmed by several independent studies. It remains unclear if $E Z H 2$ acts as a CSC-specific oncogene in some tumors (brain and prostate) and as a 'classical' oncogene in others (breast). Indeed, EZH2 participates to the silencing of several tumor suppressor genes, like E-cadherin [15], which play a crucial role for both differentiated tumor cells and CSCs. Global epigenetic profiles of cancer subpopulations [16], if applied with the above mentioned caveats, could help dissecting this double role of EZH2. For example, an interesting experiment would be to compare the epigenetic profile of CSCs and differentiated cancer cells, in presence of EZH2 inhibition.

Whether EZH2 is a 'classical' oncogene or a CSC-specific one, our hope is that new discoveries on cancer biology and new therapeutic options will emerge from the study of epigenetic modifications in CSCs.

\section{Financial \& competing interests disclosure}

The author has no relevant affiliations or financial involvement with any organization or entity with a financial interest in or financial conflict with the subject matter or materials discussed in the manuscript. This includes employment, consultancies, honoraria, stock ownership or options, expert testimony, grants or patents received or pending, or royalties.

No writing assistance was utilized in the production of this manuscript.

\section{Bibliography}

1 Surface LE, Thornton SR, Boyer LA: Polycomb group proteins set the stage for early lineage commitment. Cell Stem Cell, 7 , 288-298 (2010).

2 Aoki R, Chiba T, Miyagi S et al.: The polycomb group gene product Ezh2 regulates proliferation and differentiation of murine hepatic stem/ progenitor cells. J. Hepatol. 52, 854-863 (2010).

3 Varambally S, Dhanasekaran SM, Zhou M et al.: The polycomb group protein EZH2 is involved in progression of prostate cancer. Nature 419, 624-629 (2002).

4 Crea F, Mathews LA, Farrar WL, Hurt EM: Targeting prostate cancer stem cells. Anticancer Agents Med. Chem. 9, 1105-1113 (2009).

5 Suva ML, Riggi N, Janiszewska M et al.: $\mathrm{EZH} 2$ is essential for glioblastoma cancer stem cell maintenance. Cancer Res. 69, 9211-9218 (2009).
6 Orzan F, Pellegatta S, Poliani L et al:: Enhancer of zeste 2 (EzH2) is up-regulated in malignant gliomas and in glioma stem-like cells. Neuropathol. Appl. Neurobiol. DOI: doi: 10.1111/j.1365-2990.2010.01132 (2010) (Epub ahead of print).

7 Crea F, Hurt EM, Farrar WL: Clinical significance of Polycomb gene expression in brain tumors. Mol. Cancer 9, 265 (2010).

8 Wang H, Ouyang Y, Somers WG, Chia W, Lu B: Polo inhibits progenitor self-renewal and regulates Numb asymmetry by phosphorylating Pon. Nature 449, 96-100 (2007).

9 Yu J, Yu J, Rhodes DR et al:: A polycomb repression signature in metastatic prostate cancer predicts cancer outcome. Cancer Res. 67, 10657-10663 (2007).

10 Berezovska OP, Glinskii AB, Yang Z, Li XM, Hoffman RM, Glinsky GV: Essential role for activation of the Polycomb group (PcG) protein chromatin silencing pathway in metastatic prostate cancer. Cell Cycle 5, 1886-1901 (2006).
11 Hurt EM, Farrar WL: Cancer stem cells: the seeds of metastasis? Mol. Interv. 8, 140-142 (2008).

12 Yasuda H, Soejima K, Watanabe H et al.: Distinct epigenetic regulation of tumor suppressor genes in putative cancer stem cells of solid tumors. Int. J. Oncol. 37, 1537-1546 (2010).

13 Hurt EM, Kawasaki BT, Klarmann GJ, Thomas SB, Farrar WL: CD44+ CD24(-) prostate cells are early cancer progenitor/stem cells that provide a model for patients with poor prognosis. Br. J. Cancer $98,756-765$ (2008).

14 Dick JE: Looking ahead in cancer stem cell research. Nat. Biotechnol. 27, 44-46 (2009).

15 Cao Q, Yu J, Dhanasekaran SM et al:: Repression of E-cadherin by the polycomb group protein EZH2 in cancer. Oncogene 27, 7274-7284 (2008).

16 Mathews LA, Hurt EM, Zhang X, Farrar WL: Epigenetic regulation of $\mathrm{CpG}$ promoter methylation in invasive prostate cancer cells. Mol. Cancer 9, 267 (2010). 